\title{
VII. Spektralproblem und Komplexitätstheorie
}

von Claude-André Christen

\section{Einleitung und Uebersicht}

Ein Merkmal der modernen Axiomensysteme ist ihre Nicht-Kategorizität, d.h. die Tatsache, dass solche systeme im allgemeinen nicht eine einzige Struktur, sondern eine ganze Klasse von Strukturen charakterisieren. Obwohl der Zweck eines Systems ausdrücklich darin besteht, die gemeinsamen Eigenschaften ihrer Modelle darzustellen, bleibt das Interesse nach der Klassifizierung dieser Modelie weiterhin bestehen.

Die einfachste Eigenschaft einer Struktur ist wohl ihre Mächtigkeit; die Frage nach den Mächtigkeiten der Modelle einer axiomatischen Theorie liegt also nahe, und lässt sich manchmal genau beantworten. Allgemein bekannt sind die Beispiele der Körper, der Körper einer gegebenen Charakteristik, der Schiefkörper und der Booleschen Algebren: Die Ordnung eines endlichen Kärpers ist Potenz einer Primzahl (nämlich der Körper-Charakteristik), und umgekehrt gibt es zu jeder Primzahipotenz einen Kärper dieser Ordnung; weiter gitt es Kärper jeder unendlichen Mächtigkeit. Nach dem Satz von Wedderburn gibt es keine endliche, nichtkommutative Schiefkörper; es gibt aber nicht-kammutative Schiefkörper jeder unendlichen Mächtigkeit. Schliesslich sind die Mächtigkeiten der Booleschen Algebren genau die Zweierpotenzen und die unendlichen Mächtigkeiten.

In anderen Fällen ist das Problem ungelöst: Man weiss zum Beispiel heutzutage nicht, für welche Mächtigkeiten es einfache Gruppen dieser Ordnung gibt. Ebenfalls weiss man auch nicht, welche Mächtigkeiten als Ordnung einer nicht-auflösbaren Gruppe auftreten; allein der Nachweis der alten Burnside-Vermutung (laut welcher alle nicht-aufläsbaren Gruppen gerade Ordnung haben) braucht ungeheuren Aufwand.

An sich hat die besprochene Mächtigkeitsfrage keine allzu grosse Bedeutung; sie wird deshalb bei einer Theorie auch nur dann in Betracht gezogen, wenn sie in Verbindung mit tiefer liegenden Struktureigenschaften auftritt.

Eine ganz andere Bedeutung hat aber folgendes Problem, wo das Interesse von den einzelnen Theorien zu den dadurch definierbaren Mächtigkeitsklassen verschoben wird: Was sind überhaupt die möglichen Mächtigkeitsklassen der Modelle einer axiomatischen Theorie? Natürlich ist dabei 
eine Präzisierung des Wortes "Theorie" notwendig. Erstaunlicherweise wurde dieses Problem erst durch (Scholz 1952) gestellt, und zwar für Theorien, die in der Prädikatenlogik erster Stufe (mit Eleichheit) formulierbar sind.

Besondere Eigenschaften der Prädikatenlogik erster Stufe erlauben übrigens eine engere Formulierung des Froblems:

Zunächst ist es klar, dass das Problem trivial wird, falls man das Gleichheitszeichen nicht zulässt: Hat dann eine Theorie überhaupt ein Model1, so auch ein Modell jeder grösseren Mächtigkeit.

Auf Grund des Satzes von Löwenheim-Skolem hat eine abzählbare Theorie erster Stufe entweder keine unendichen Modelle oder Modelle jeder unendlichen Mächtigkeit. Weiter folgt aus dem Kompaktheitssatz, dass es eine endliche Schranke für die Mächtigkeiten der Modelle einer Theorie gibt, falls diese Theorie kein unendliches Modell besitzt. Es genügt also, die Frage für die endlichen Mächtigkeiten zu stellen.

Lässt man Systeme mit unendlich vielen Axiomen zu, so wird das problem wiederum trivial: Jede Teilmenge der Menge der positiven ganzen Zahlen ist in dieser Weise darstellbar, denn jede endliche Mächtigkeit ist schon mit einer Gleichheitsformel ohne freie Variable charakterisierbar; die negierte Formel schliesst dann gerade diese Mächtigkeit aus. Werden aber nur endlioh viele Axiome zugelassen - oder nur eines, was auf dasselbe herauskommt - so können nur noch abzählbar viele Mengen dargestellt werden.

Auf Grund dieser Reduktionen definiert man das Spektrum einer logischen Formel als die Menge der Mächtigkeiten ihrer endlichen Modelle. Eine Menge heisst dann Spektrum erster Stufe, falls es eine Formel erster Stufe gibt, deren Spektrum sie ist. Das spektralproblem lautet nun: Welche Mengen sind Spektren erster Stufe? (Zur Abkürzung wird von nun an unter "Spektrum" immer "Spektrum erster Stufe" gemeint.)

Es sei noch ausdrücklich hervargehoben, dass dieses Problem einen extensionalen Standpunkt voraussetzt: Im Falle der Zweierpotenzen zum Beispiel ist es gléichgultig, ob die Menge als Spektrum oines Axiomensystems für die Booleschen Algebren oder für die Kärper der Charakteristik 2 - oder durch irgend ein anderes passendes system - dargestellt wird: Es kommt nur darauf an, dass mindestens eine solohe Charakterisierung existiert.

Es stellt sich tald heraus, dass die spektren schon recht komplizierte 
Mengen sein kännen. Das Spektrum der Theorie der nicht-zyklischen Gruppen besteht zum Beispiel genau aus den Zahlen, welche durch eine von 1 verschiedene Quadratzahl, oder durch zwei Primzahlen teilbar ist, deren eine kongruent 1 modulo die andere ist. Es gibt genau dann eine nichtabelsche Gruppe der endlichen Ordnung $n$, wenn $n$ durch eine von 1 verschiedene Kubikzahl oder durch $\mathrm{pq}^{2}$ teilbar ist, wobei $p$ und q prim sind, und $q^{2}$ kongruent 1 modulo $p$ ist (siehe Pazderski 1959, wo nooh andere Beispiele behandelt werden).

Es ist sogar einfacher, Mengen anzugeben, die Spektren sind, als Mengen, die es nicht sind - eine Berechtigung dieser unmathematischen Behauptung ist die Tatsache, dass alle bekannten Beispiele von Mengen, die keine Spektren sind, mehr ader weniger auf Diagonalisierung beruhen. Wie schlecht die Struktur der Spektren noch verstanden wird, zeigt die einfache, doch ungelöste Frage (Asser 1956): Ist die Klasse der Spektren gegenüber Komplementbildung abgeschlossen? (Es ist dagegen leicht nachzuweisen, dass sie gegenüber Vereinigung und Durchschnitt abgeschlossen ist.)

Im zweiten Paragraphen werden Beispiele angegeben, welche die Kompliziertheit der Spektren veranschaulichen, und zudem einen Vorgeschmack für die später benützte Darstellungsmethode angeben:

Obwahl alle Spektren primitiv rekursiv sind, gibt es ein Spektrum, dessen Aufzählungsfunktion schliesslich stärker als jede primitiv rekursive Funktion wächst. Die Modelle der gewählten zugehörigen Formel stellen Anfangsstücke des Graphen einer Modifikation der Ackermann-Funktion dar.

Es gibt aber sogar zu jeder rekursiven Funktion ein Spektrum, dessen Aufzählungsfunktion stärker als diese Funktion wächst. Diese Behauptung lässt sich aus der Tatsache ableiten, dass die konstruktiv arithmetischen Mengen Spektren sind. Daraus lässt sich ebenfalls beweisen, dass es nicht rekursiv entscheidbar ist, ob ein Spektrum leer ist oder nicht (dies wurde schon von Trachtenbrot 1950 bewiesen); das Problem hat sogar den höchsten Unentscheidbarkeitsgrad unter den rekursiv aufzählbaren Problemen (Büchi 1962). Analog ist das Unendlichkeitsproblem für Spektren $\Pi \Sigma$-vollständig bezüglich Turing-Reduzibilität.

Es ist jedoch klar, dass die Spektren nur eine beschränkte Komplexität aufweisen kännen, denn es gibt nur $2 n^{r}$ r-stellige Prädikate auf einem n-elementigen Bereich. Deshalb ist die Zugehörigkeit der Zahl $n$ zu einem 
Spektrum für ein geeignetes $c$ in höchstens $2^{n^{C}}$ Schritte auf einer Turing-Maschine entscheidbar. Als Folgerung erweist sich die Klasse der Spektren als eine Teilklasse der Klasse der im Kalmárschen Sinne elementaren Funktionen; (Asser 1956) bewies schon, dass die Inklusion echt ist.

Im dritten Paragraphen wird das Hauptergebnis von (Jones \& Selman 1974) bewiesen: Die Spektren stimmen mit den Mengen überein, welche durch eine schliesslich exponentiell (d.h. der Form $\lambda \times .2^{\mathrm{Cx}}$ für $0 \in \mathbb{N}$ ) beschränkte nichtdeterministische Turing-Maschine akzeptiert werden. Wird als Input nicht die dyadische Kodierung von $n$, sondern ein wort der Länge $n\left(z . B . ~ n\right.$ mal $b_{1}$ ) gebraucht, so ist die Schranke polynomial. Deshalb ergibt sich ein Zusammenhang mit dem Problem $P=N P$ : Gilt $P=N P$, so sind auch die Spektren deterministisch mit einer polynomialen Schranke auf einer Turingmaschine zu berechnen (bei Input $n$ mal $b_{1}$ ). Analog: Ist NP gegenüber Komplementbildung abgeschlossen, so auch die Klasse der Spektren. Ein Beweis, dass die Klasse der Spektren gegenüber Komplementbildung nicht abgeschlassen ist, ist also mindestens so schwer, wie ein Beweis, dass NP gegenüber Kamplementbildung nicht abgeschlossen ist; und ein Beweis, dass es ein Spektrum gibt, das durch keine deterministische exponentiell-beschränkte Turingmaschine berechenbar ist, mindestens so schwer, wie ein Beweis von P+NP.

Im vierten Paragraphen werden die Mengen aus NP und die endlich axiomatisierbaren projektiven Klassen von Strukturen von endichem Typus in Verbindung gebracht. Der gewonnene Satz ist eine Verallgemeinerung des früheren Hauptsatzes, betrifft allerdings nur die endichen Strukturen einer solchen Klasse: Mittels einer gewissen Kodierung fallen diese Klassen von endichen Strukturen und die Mengen von NP zusammen. Man achte doch darauf, dess der hier angenommene Standpunkt nicht derjenige der Modelltheorie ist, sondern eher derjenige der finiten Mathematik (mit einem naiven Begriff des Endlichen).

Es gibt zum Beispiel ein nicht-deterministisches polynomiales Verfahren, welches die endlichen zyklischen Gruppen akzeptiert und die endlichen nicht-zyklischen Gruppen verwirft. Dies entspricht hier der Tatsache, dass es eine endlich axiomatisierbare projektive klasse gibt, deren endliche Strukturen genau die endlichen zyklischen Gruppen sind, obwohl weder die Klasse der endlichen zyklischen Gruppen noch die ganze Klasse der zyklischen Gruppen eine projektive Klasse bilden.

Endlich werden noch am Schluss der Arbeit Ergebnisse über Spektren 
häherer Stufe aufgezählt; für Beweise wird auf die zitierte Lïteratur hingewiesen.

\section{Beispiele von Spektren}

Zunächst wird kurz die Sprache der Prädikatenlogik erster Stufe beschrieben, sowie die Begriffe "Interpretation" und "Modell" erläutert. Dis Grundzeichen der Prädikatenlogik erster Stufe sind die (logischen) Zeichen $v, \wedge, \neg, \exists, \forall,(),,=$ und die (nicht-logischen) Zeichen aus $Z=V$ U FZ UPZ: $V=\left\{v_{i} \mid i \in \mathbb{N}\right\}$ ist die Menge der Individuenvariablen; $F Z=\left\{f_{i}^{j} \mid i, j \in \mathbb{N}\right\}$ ist die Menge der Funktionszeichen; $P Z=\left\{p_{i}^{j} \mid i, j \in \mathbb{N}\right\}$ ist die Menge der Prädikatszeichen.

Aus diesen Grundzeichen werden Ausdrücke - d.h. Folgen von Zeichen gebildet. Die Concatenation von Folgen wird einfach durch Hintereinanderschreiben der Folgen bezeichnet. Bedeutung wird nur den folgenden drei Klassen $T, A$ und $F$ von Ausdrücken zuerkannt:

Die Menge $T$ der Terme ist die kleinste Menge, welche die Falgen der Länge 1 , bestehend aus einem Zeichen aus $V$, enthält, und welche für $i, j \in \mathbb{N}$ die Concatenation von $f_{i}^{j}$ mit den $j$ Termen $t_{1}, \ldots, t_{j}$ enthält. Die Zeichen $f_{i}^{j}$ heissen deshalb "j-stellige" Zeichen; der Lesbarkeit halber wird - auch im Falle $j=0$ - die Folge $f_{i}^{j} t_{1} \ldots t_{j}$ mit $f_{i}^{j}\left(t_{1}, \ldots, t_{j}\right)$ bezeichnet.

Die Menge A der Atomformeln ist die Menge der Ausdrücke der Form $t_{1}=t_{2}$ und $p_{i}^{j}\left(t_{1}, \ldots, t_{j}\right)$ für $i, j \in \mathbb{N}$ und $t_{1}, \ldots, t_{j} \in T$ (mit derselben Bezeichnungskonvention wie oben; die $p_{i}^{j}$ heissen auch j-steliig).

Die Menge $F$ der Formeln ist die kleinste Menge, welche A enthält und mit $f_{1}, f_{2} \in F$ und $v \in V$ auch die Ausdrücke $\left(f_{1} \vee f_{2}\right),\left(f_{1} \wedge f_{2}\right), \neg f_{1},(\exists v) f_{1},(\forall v) f_{1}$ enthält.

Es lässt sich beweisen, dass jeder Term (sowie jede Formel) gemäss diesen Regeln auf eine einzige Weise gebildet ist.

-Formeln der Form $\left(Q_{1} v_{i}\right) \ldots\left(Q_{k} v_{i_{k}}\right) f$, wobei jedes $Q_{j}$ ein Quantorzeichen $\forall$ oder $\exists$ bezeichnet und $f$ keine Quantorzeichen enthält, heissen pränex. $f$ ist die Matrix der Formel und $\left(Q_{1} v_{i}\right) \ldots\left(Q_{k} v_{i_{k}}\right)$ ihr Präfix. Bekanntlich gibt es zu jeder Formel eine dazu äquivalente pränexe formel. Eine Formel heisst abgeschlossen, wenn jede Okkurenz einer Individuenvariablen $v$ auch Okkurenz einer Teilformel von $f \operatorname{der}$ Form ( $\exists v$ ) f' oder 
$(\forall v) f^{\prime}$ ist.

Eine Struktur für eine Formel f der Prädikatenlogik erster Stufe ist ein Paar <M,I>, wabei $M$ eine nicht-leere Menge ist, und $I$ eine fü die in $f$ vorkommenden Zeichen aus $Z$ definierte Funktion ist, welche folgende Eigenschaften besitzt:

(a) für $i \in \mathbb{N}$ ist $I\left(v_{i}\right)$ eine Funktion von $M^{\mathbb{N}}$ nach $M$; dabei ist $\left[I\left(v_{i}\right)\right](c)=c_{i}$ für $c \in M^{\mathbb{N}}$

(b) für $i, j \in \mathbb{N}$ ist $I\left(f_{i}^{j}\right)$ eine Funktion von $M^{j}$ nach $M$;

(c) für $i, j \in \mathbb{N}$ ist $I\left(p_{i}^{j}\right)$ eine Funktion von $M^{j}$ nach $2=\{0,1\}$. Bemerkung: $M^{0}$ ist $\{\theta\}$.

Aus I wird induktiv eine Interpretation J der Terme und Formeln, die aus diesen Zeichen gebildet werden, wie folgt eindeutig definiert:

(1) für $i, j \in \mathbb{N}, t_{1}, \ldots, t_{j} \in T$ und $c \in M^{\mathbb{N}}$ ist $J\left(f_{i}^{a}\right)(c)=I\left(f_{i}^{a}\right)(\emptyset)$ und $J\left(f_{i}^{j+1}\left(t_{1}, \ldots, t_{j+1}\right)(c)=I\left(f_{i}^{j+1}\right)\left(J\left(t_{1}\right)(c), \ldots, J\left(t_{j+1}\right)(c)\right) ;\right.$

(2) für $i, j \in \mathbb{N}, t_{1}, \ldots, t_{j} \in T$ und $o \in M^{\mathbb{N}}$ ist $J\left(p_{i}^{0}\right)(c)=I\left(p_{i}^{0}\right)(\varnothing)$ und $J\left(p_{i}^{j+1}\left(t_{1}, \ldots, t_{j+1}\right)\right)(c)=I\left(p_{i}^{j+1}\right)\left(J\left(t_{1}\right)(c), \ldots, J\left(t_{j+1}\right)(c)\right) ;$

(3) für $t, t^{\prime} \in T$ und $c \in M^{\mathbb{N}}$ ist $J\left(t=t^{\prime}\right)(c)=\left\{\begin{array}{l}1 \text { falls } J(t)(c)=J\left(t^{\prime}\right)(c) \\ 0 \text { sonst; }\end{array}\right.$

für $f, f^{\prime} \in F, i \in \mathbb{N}$ und $c \in M^{\mathbb{N}}$ ist:

(4) $J\left(f \vee f^{\prime}\right)(c)=\max \left(J(f)(c), J\left(f^{\prime}\right)(c)\right)$

(5) $J\left(f \wedge f^{\prime}\right)(c)=\min \left(J(f)(c), J\left(f^{\prime}\right)(c)\right)$

(6) $J(\neg f)(c)^{*}=1-J(f)(c)$

(7) $J\left(\left(\exists v_{i}\right) f\right)(c)=\max _{m \in M} J(f)\left(\left.c\right|_{m} ^{c}\right)$

(8) $J\left(\left(\forall v_{i}\right) f\right)(c)=\min _{m \in M} J(f)\left(\left.c\right|_{m} ^{c_{i}}\right)$

(dabei ist $\left.c\right|_{m} ^{c_{i}}(k)=\left\{\begin{array}{l}c_{k} \text { falls } k \neq i \\ m \text { sonst }\end{array}\right.$ )

Eine Struktur <M, I> ist ein Modell von $f$, falls es für die von I erzeugte Interpretation ein $\in M^{\mathbb{N}}$ gibt, mit $J(f)(c)=1$. Eine Formel heisst $k$-elementig erfüllbar, falls sie ein Modell $\langle M, I\rangle$ mit $\overline{\bar{M}}=k$ besitzt. 
Das Spektrum einer Formel f ist die Menge der positiven Zahlen $n$, für welche $f$-elementig erfülbar ist.

Als erstes Beispiel werden die Spektren von Gleichheitsformeln untersucht - das sind die Formeln, die weder Funktions- noch Prädikatszeichen enthalten. Wie man leicht aus den angegebenen Definitionen ableiten kann, ist $\mathbb{N}$ das Spektrum der Formel $v_{0}=v_{0}$ und die leere Menge das Spektrum der Farmel $\neg v_{0}=v_{0}$ (die kurz $v_{0}+v_{0}$ geschrieben wird). Das spektrum der Formel $\left(\exists v_{0}\right)\left(\exists v_{1}\right) v_{0} \neq v_{1}$ (oder von $v_{0} \neq v_{1}$, was für die Erfüllbarkeit auf dasselbe herauskommt) besteht aus allen ganzen Zahlen, die grösser als 1 sind; dasjenige von $\left(\forall v_{0}\right)\left(\forall v_{1}\right) v_{1}=v_{0}$ oder dasjenige von $\left(\exists v_{0}\right)\left(\forall v_{1}\right) v_{1}=v_{0}$ dagegen aus der einzigen Zah1 1. Allgemeiner besteht das Spektrum der Formel.

$$
\left.\left(\exists v_{0}\right) \ldots\left(\exists v_{k}\right)\left(\ldots\left(v_{0} \neq v_{1} \wedge v_{0} \neq v_{2}\right) \wedge v_{0} \neq v_{3}\right) \ldots \wedge v_{k-1} \neq v_{k}\right) \text {, }
$$

welche durch $\left(\exists v_{0}\right) \ldots\left(\exists v_{k}\right) \bigwedge_{0 \leq i<j<k} v_{i}{ }^{\dagger} v_{j}$ abgekürzt wird, aus den Zahlen, die grösser als $k$ sind, und J̈as S̄pektrum der Formel

$$
\left.\left(\forall v_{0}\right) \ldots\left(\forall v_{k+1}\right)\left(\ldots\left(v_{0}=v_{1} v v_{0}=v_{2}\right) v v_{0}=v_{3}\right) \ldots v v_{k}=v_{k+1}\right) \text {, }
$$

welche durch $\left(\forall v_{0}\right) \ldots\left(\forall v_{k+1}\right) \bigvee_{0 \leq i<j \leq k+1} v_{i}=v_{j}$ abgekürzt wird, aus den Zahlen, die kleiner als $k+2$ sind; dasselbe Spektrum hat die formel

$$
\left(\exists v_{0}\right) \ldots\left(\exists v_{k}\right)\left(\forall v_{k+1}\right) \bigvee_{0 \leq i \leq k} v_{k+1}=v_{i} \text {. }
$$

Folglich besteht das spektrum von

$$
\left(\exists v_{0}\right) \ldots\left(\exists v_{k}\right)\left(\forall v_{k+1}\right)\left(\bigwedge_{0 \leq i<j \leq k} v_{i} \neq v_{j} \wedge \bigvee_{0 \leq i \leq k} v_{k+1}=v_{i}\right)
$$

aus der einzigen Zah1 $k+1$.

Eine beliebige endliche Menge ist also Spektrum einer Gleichheitsformel: Nämlich Spektrum der Disjunktion von Formeln der zuletzt erwähnten Form, die die Elemente der Menge charakterisieren. Das Spektrum der Negation dieser Formel ist die komplementäre Menge; folglich ist auch jede koendliche Menge Spektrum einer Gleichheitsformel.

Ungekehrt gilt aber auch, dass die Spektren der Gleichheitsformeln genau die endlichen und koendlichen Mengen sind: Da diese Formeln weder Funktions- noch Prädikatszeichen enthalten, sind die Klassen der Mächtigkeiten der Modelle einer abgeschlossenen Formel und ihrer Negation komplementär. Nach dem Satz von Löwenheim hat also genau eine davon unendliche Modelle; nach dem Kompaktheitssatz hat aber dann die andere Formel ein endiches spektrum.

Dieser Beweis lässt sich nicht auf den allgemeinen Fall erweitern, denn die Formeln $\left(\exists v_{0}\right) p_{0}^{1}\left(v_{0}\right)$ und $\neg\left(\exists v_{0}\right) p_{0}^{1}\left(v_{0}\right)$ sind schon beide in beliebi- 
gen Bereichen erfüllbar. Trotzdem sind die Spektren der monadischen Formeln (d.h. der Formeln, die keine Funktionszeichen und keine mehrstellige Prädikatszeichen enthalten) wieder genau die endlichen und die koendlichen Mengen - ein Resultat, das auch auf (Löwenheim 1915) zurückgeht. Der Beweis dieser Behauptung beruht auf einem sogenannten Quantoreneliminationsverfahren und kann z.B. in (Ackermann 1954) gefunden werden. Gewisse Spektren können aber mit monadischen Formeln dargestellt werden, die viel kürzer als jede zugehörige Gleichheitsformel sind: Das Spektrum der Formel $f_{k}$ : $\left(\forall v_{0}\right)\left(\forall v_{1}\right)\left(v_{0}=v_{1} v\left(\bigvee_{0 \leq i<k}\left(p_{i}^{1}\left(v_{0}\right) \wedge \neg p_{i}^{1}\left(v_{1}\right)\right) \vee \bigvee_{0 \leq i<k}\left(\neg p_{i}^{1}\left(v_{0}\right) \wedge p_{i}^{1}\left(v_{1}\right)\right)\right)\right)$ besteht aus allen Zahlen, die kleiner oder gleich $2^{k}$ sind; eine Gleichheitsformel mit diesem Spektrum hat aber eine Länge grösser als $2^{k}$; die Länge der Formeln $f_{k}$ wächst dagegen nur linear mit $k$.

Sobald ein einziges 1-stelliges Funktionszeichen oder ein einziges 2stelliges Prädikatszeichen in einer Formel enthalten ist, so kann das Spektrum der Formel schon unendlich sein und unendliches Komplement haben. Das Spektrum von

$$
\left(\forall v_{0}\right)\left(f_{0}^{1}\left(v_{0}\right) \neq v_{0} \wedge f_{0}^{1}\left(f_{0}^{1}\left(v_{0}\right)\right)=v_{0}\right)
$$

besteht genau aus den geraden Zahlen. Nun kann eine Funktion immer durch ihren Graphen dargestellt werden: So hat zum Beispiel die Konjunktion der drei Formeln

$\left(\forall v_{0}\right)\left(\exists v_{1}\right) p_{0}^{2}\left(v_{0}, v_{1}\right)$

$\left(\forall v_{0}\right)\left(\forall v_{1}\right)\left(\forall v_{2}\right)\left(\neg\left(p_{0}^{2}\left(v_{0}, v_{1}\right) \wedge p_{0}^{2}\left(v_{0}, v_{2}\right)\right) v_{1} v_{1}=v_{2}\right)$

$\left(\forall v_{0}\right)\left(\neg p_{0}^{2}\left(v_{0}, v_{0}\right) \wedge\left(\exists v_{1}\right)\left(p_{0}^{2}\left(v_{0}, v_{1}\right) \wedge p_{0}^{2}\left(v_{1}, v_{0}\right)\right)\right)$

dasselbe Spektrum wie die obige Formel. In dieser Weise kann man übrigens jeder formel eine neue formel mit demselben Spektrum zuordnen, welche keine Funktionszeichen enthält.

Nebenbei sei auch bemerkt, dass es Formeln mit einem einzigen 1-stelligen Funktionszeichen bzw. 2-stelligen Prädikatszeichen gibt, die erst abzählbar erfüllbar sind - was bei monadischen Formeln nicht der fall ist. Solche sind zum Beispiel

$\left(\exists v_{0}\right)\left(\forall v_{1}\right)\left(\forall v_{2}\right)\left(\left(f_{0}^{1}\left(v_{1}\right) \neq v_{0} \wedge f_{0}^{1}\left(v_{1}\right) \neq v_{1}\right) \wedge\left(f_{0}^{1}\left(v_{1}\right) \neq f_{0}^{1}\left(v_{2}\right) v v_{1}=v_{2}\right)\right)$ und

$\left(\forall v_{0}\right)\left(\exists v_{1}\right)\left(\forall v_{2}\right)\left(\left(\neg p_{0}^{2}\left(v_{0}, v_{0}\right) \wedge p_{0}^{2}\left(v_{0}, v_{1}\right)\right) \wedge\left(\neg p_{0}^{2}\left(v_{1}, v_{2}\right) v_{0}^{2}\left(v_{0}, v_{2}\right)\right)\right)$

Wie leicht einzusehen ist, sind die spektren von existentiellen (bzw. abgeschlossenen universellen) pränexen Formeln, die keine Funktions- 
zeichen enthalten, Reststücke (bzw. Anfangsstücke) der Menge der positiven Zahlen; die angegebenen Beispiele von Gleichheitsformeln zeigen, dass alle Rest- und Anfangsstücke vorkommen. Ebenfalls sind die Spektren von pränexen Formeln ohne Funktionszeichen, in welchen die Existentialquantoren sämtichen Universalquantoren vorangehen (wie in $\left.\left(\exists v_{0}\right)\left(\forall v_{1}\right) v_{1}=v_{0}\right)$ genau die endlichen und koendlichen Mengen. Dagegen lässt sich aber beweisen, dass jedes Spektrum das Spektrum einer pränexen Formel (erster Stufe) ohne Funktionszeichen ist, in welcher die Universalquantoren sämtlichen Existentialquantoren vorangehen (Skolemsche Normalform bez. Erfüllbarkeit). Dies entspricht der aussagenlogischen Reduktion auf die konjunktive Normalform in II: Jedes Modeli der Formel

$$
\left(v_{v_{0}}\right)\left(\exists v_{1}\right)\left(\forall v_{2}\right) p_{0}^{3}\left(v_{0}, v_{1}, v_{2}\right)
$$

lässt sich zu einem Modell der Formel.

$$
\left(\forall v_{0}\right)\left(\forall v_{1}\right)\left(\forall v_{2}\right)\left(\exists v_{3}\right)\left(\left(\neg p_{1}^{2}\left(v_{0}, v_{1}\right) v_{0}^{3}\left(v_{0}, v_{1}, v_{2}\right)\right) \wedge p_{1}^{2}\left(v_{0}, v_{3}\right)\right)
$$

erweitern, und jedes Modell der zweiten Formel induziert ein Modell der ersten Formel. Sind Funktionszeichen zugelassen, so darf man sich entsprechend auf universelle prënexe Formeln einschränken: Das Spektrum von $\quad\left(\forall v_{0}\right)\left(\forall v_{1}\right) p_{0}^{3}\left(v_{0}, f_{0}^{1}\left(v_{0}\right), v_{1}\right)$

ist dasselbe wie dasjenige der obigen Formel.

In den nachfolgenden Beispielen werden Anfangsstücke von arithmetischen Relationen in einem gewissen Sinne durch formeln dargestellt. Um die Formulierung zu vereinfachen, werden jetzt sogenannte geordnete Modelle betrachtet: Ein geordnetes Modell von $f$ ist ein Modell, dessen Trägermenge ein Anfangsstück von $\mathbb{N}-d . h$. eine Menge $\{0,1, \ldots, n\}$ - ist und bei dem das Prädikatszeichen $p_{0}^{2}$ durch < interpretiert wird. Falls das zeichen $p_{0}^{2}$ nicht in $f$ vorkommt, so ist die letztere Bedingung leer. Somit gibt es zu jedem Spektrum S eine Formel, welche genau dann ein n-zehliges geordnetes Modell besitzt, wenn $n$ zu S gehört. Hiervon gilt auch die Umkehrung: Sei $f$ eine Formel und $T$ die Menge derjenigen $n$, zu denen es ein n-zahliges geordnetes Modell gibt; dann ist $T$ ein Spektrum.

Es sei nämlich $f^{\prime}$ die Konjunktion von $f$ und einer Formel, welche ausdrückt, dass $p_{0}^{2}$ eine Ordnungsrelation ist. Dann ist einmal jedes geordnete Modell von $f$ auch ein Modell von f'; umgekehrt ist jedes endliche Modell von $f^{\prime}$ 'isomorph einem endlichen geordneten Modell von $f$ ' und 
somit auch einem endlichen Modell von $f$.

Definition Die arithmetische Relation $R$ wird durch die Formel $f$ dargestellt, wenn folgende Bedingungen erfültt sind:

(1) Zu jeder positiven ganzen Zahl $n$ gibt es ein n-zahliges geordnetes Modell von $f$.

(2) Es gibt ein Prädikatszeichen $p_{i}^{s}$, dessen Interpretation in jedem endlichen geordneten Modell <M, I> von $f$ mit der Einschränkung von $R$ auf M übereinstimmt.

Lemma Der Graph der Funktion a, welche durch das Rekursionsschema

$$
\begin{cases}a(0, y, z) & =z+1 \\ a(x+1, y, 0) & =y \\ a(x+1, y, z+1) & =a(x, y, a(x+1, y, z))\end{cases}
$$

definiert wird, wird durch eine Formel erster stufe dargestellt.

Beweis $f$ sei die Konjunktion folgender Formeln (dabei ist $\left(f_{1} \leftrightarrow f_{2}\right.$ ) eine Abkürzung von $\left.\left(\neg f_{1} \vee f_{2}\right) \wedge\left(f_{1} \vee \neg f_{2}\right)\right)$ :

(1) $\left(\forall v_{0}\right) \neg p_{0}^{2}\left(v_{0}, v_{0}\right)$

(2) $\left(\forall v_{0}\right)\left(\forall v_{1}\right)\left(v_{0}=v_{1} v\left(p_{0}^{2}\left(v_{0}, v_{1}\right) v p_{0}^{2}\left(v_{1}, v_{0}\right)\right)\right)$

(3) $\left(\forall v_{0}\right)\left(\forall v_{1}\right)\left(\forall v_{2}\right)\left(\neg\left(p_{0}^{2}\left(v_{0}, v_{1}\right) \wedge p_{0}^{2}\left(v_{1}, v_{2}\right)\right) v_{p_{0}}^{2}\left(v_{0}, v_{2}\right)\right)$

(4) ( $\left.\exists v_{0}\right) p_{1}^{1}\left(v_{0}\right)$

(5) $\left(\forall v_{0}\right)\left(\forall v_{1}\right)\left(\neg p_{1}^{1}\left(v_{0}\right) v \neg p_{0}^{2}\left(v_{1}, v_{0}\right)\right)$

(6) $\left(\forall v_{0}\right)\left(\forall v_{1}\right)\left(\neg p_{2}^{2}\left(v_{0}, v_{1}\right) v_{p_{0}}^{2}\left(v_{0}, v_{1}\right)\right)$

( ᄀ) $\left(\forall v_{0}\right)\left(\forall v_{1}\right)\left(\forall v_{2}\right)\left(\neg p_{2}^{2}\left(v_{0}, v_{1}\right) \vee\left(\neg p_{0}^{2}\left(v_{0}, v_{2}\right) \vee \neg p_{0}^{2}\left(v_{2}, v_{1}\right)\right)\right)$

(8) $\left(\forall v_{0}\right)\left(\exists v_{1}\right)\left(p_{1}^{1}\left(v_{0}\right) v_{p}^{2}\left(v_{1}, v_{0}\right)\right)$

(g) $\left(\forall v_{0}\right)\left(\forall v_{1}\right)\left(\forall v_{2}\right)\left(\forall v_{3}\right)\left(\neg p_{1}^{1}\left(v_{0}\right) v\left(p_{2}^{2}\left(v_{2}, v_{3}\right) \longleftrightarrow p_{3}^{4}\left(v_{0}, v_{1}, v_{2}, v_{3}\right)\right)\right)$

(10) $\left(\forall v_{0}\right)\left(\forall v_{1}\right)\left(\forall v_{2}\right)\left(\forall v_{3}\right)\left(\left[\neg p_{1}^{1}\left(v_{2}\right) v_{p_{1}^{1}}^{1}\left(v_{0}\right)\right) v\left(p_{3}^{4}\left(v_{0}, v_{1}, v_{2}, v_{3}\right) \longleftrightarrow v_{3}=v_{1}\right)\right)$

$(11)\left(\forall v_{0}\right)\left(\forall v_{1}\right)\left(\forall v_{2}\right)\left(\forall v_{3}\right)\left(\forall v_{4}\right)\left(\forall v_{5}\right)\left(\left(\neg p_{2}^{2}\left(v_{4}, v_{2}\right) v \neg p_{2}^{2}\left(v_{5}, v_{0}\right)\right) v\right.$ $\left(p_{3}^{4}\left(v_{0}, v_{1}, v_{2}, v_{3}\right) \longleftrightarrow\left(\exists v_{6}\right)\left(p_{3}^{4}\left(v_{0}, v_{1}, v_{4}, v_{6}\right) \wedge p_{3}^{4}\left(v_{5}, v_{1}, v_{6}, v_{3}\right)\right)\right)$

Es lässt sich sofort prüfen, dass für jedes $n \in \mathbb{N}$ die Formel $f$ erfüllt wird, falls die Zeichen $p_{0}^{2}, p_{1}^{1}, p_{2}^{2}$ und $p_{3}^{4}$ durch die Einschränkungen der Kleinerrelation, der charakteristischen Relation von 0 , der Nachfolgerrelation und des Graphen der Funktion a auf $n+1=\{0,1, \ldots, n\}$ interpre- 
tiert werden.

Sei weiter <M, I> ein geordnetes endliches Modell von $f$, d.h. M ist ein Anfangsstück von $\mathbb{N}$, und $I\left(p_{0}^{2}\right)$ stimmt mit der Einschränkung von < auf $M$ überein. Mit Induktion lässt sich dann aus (4) und (5), bzw. (6)-(8), (9)-(11) beweisen, dass $I\left(p_{1}^{1}\right)$ mit der Relation $\lambda x \cdot x=0$ auf $M, b z w$. I $\left(p_{2}^{2}\right)$ mit der Einschränkung der Nachfolgerrelation $\lambda x y \cdot y=x+1$ auf $M$ und (da $a$ monoton ist im 3. Argument) $I\left(\mathrm{p}_{3}^{4}\right)$ mit der Einschränkung des Graphen der Funktion a auf M übereinstimmt.

Mit dem Graphen der Funktion a wird auch der Graph der Funktion $\lambda x \cdot a(x, x, x)$ durch eine Formel dargestellt. Um dies zu sehen, fügen wir ein Prädikatszeichen $\mathrm{p}_{3}^{2}$ und das weitere Konjunktionsglied

hinzu.

$$
p_{3}^{2}\left(x_{0}, x_{1}\right) \longleftrightarrow p_{3}^{4}\left(x_{0}, x_{0}, x_{0}, x_{1}\right)
$$

Hilfssatz Der Graph der Funktion $d$ werde durch eine Formel $f$ dargestellt; dann bilden die werte van $d+1$ ein Spektrum.

Beweis Die Funktion d sei etwa zweistellig und es sei $p_{3}^{3}$ das Prädikatszeichen der Darstellung. Es sei dann $f^{\prime}$ die Konjunktion von $f$ und der Formel

$$
\left(\exists v_{0}\right)\left(\exists v_{1}\right)\left(\exists v_{2}\right)\left(p_{3}^{3}\left(v_{0}, v_{1}, v_{2}\right) \wedge \neg\left(\exists v_{3}\right) p_{0}^{2}\left(v_{2}, v_{3}\right)\right) \text {. }
$$

Ist dann M ein geordnetes Modell von $f^{\prime}$ mit der Trägermenge $\{0, \ldots, n\}$, so ist $n$ ein Funktionswert von $d$ und $(n+1)$ ist die Mächtigkeit von $M$. Umgekehrt gibt es zu jedem $n$, das Funktionswert von d ist, auch ein geordnetes Modell mit der Trägermenge $\{0, \ldots, n\}$. Da, wie gezeigt, die Menge der Zahlen $m$, zu denen es ein geordnetes Modell mit m Elementen gibt, ein Spektrum ist, so folgt die Behauptung.

Aus Lemma und Hilfssatz folgt nun unmittelbar

Satz 1 Die Werte der Funktion $\lambda x \cdot a(x, x, x)+1$ bilden ein Spektrum.

Korallar Es gibt ein Spektrum, dessen Aufzählungsfunktion schliesslich stärker als jede primitiv-rekursive Funktion wächst.

Beweis Das lässt sich wie in (Ackermann 1928) beweisen.

Das nächste Beispiel ist allgemeiner Natur:

Definition Die Klasse der konstruktiv-arithmetischen Relationen ist die kleinste Klasse, welche die Additions- und die Multiplikationsrela- 
tion enthäl, und welche gegenüber Booleschen Operationen, beschränkten Quantifikationen und expliziten Transformationen (d.h. Variablenidentifikation und -vertauschung, sowie Substitution von Konstanten) abgeschlossen ist.

(Diese Definition stammt aus (Smullyan 1961). Für Beispiele sei auf diese Arbeit verwiesen.)

Satz 2 Jede konstruktiv-arithmetische Relation ist durch eine Formel erster stufe darstellbar.

Beweis Dem induktiven Charakter der obigen Definition entsprechend wird der Beweis durch Induktion über den Aufbau der Relationen geführt. (1) Die Relationen $\lambda x \cdot x=0$ und $\lambda x y \cdot y=x+1$ werden durch die Forme in (1)-(8) des früheren Lemmas dargestellt. Folglich wird die Additionsrelation dargestelit durch die Konjunktion von (1)-(B) und der beiden Formeln:

$\left.{ }^{*}\right\} \quad\left(\forall v_{0}\right)\left(\forall v_{1}\right)\left(\forall v_{2}\right)\left(\neg p_{1}^{1}\left(v_{1}\right) v\left(p_{3}^{3}\left(v_{0}, v_{1}, v_{2}\right) \longleftrightarrow v_{0}=v_{2}\right)\right)$

**) $\left(\forall v_{0}\right)\left(\forall v_{1}\right)\left(\forall v_{2}\right)\left(\forall v_{3}\right)\left(\neg p_{2}^{2}\left(v_{3}, v_{1}\right) v\right.$

$$
\left.\left(p_{3}^{3}\left(v_{0}, v_{1}, v_{2}\right) \longleftrightarrow\left(\exists v_{4}\right)\left(p_{3}^{3}\left(v_{0}, v_{3}, v_{4}\right) \wedge p_{2}^{2}\left(v_{4}, v_{2}\right)\right)\right)\right) .
$$

(2) Analog wird die Multiplikationsrelation dargestellt durch die Konjunktion von $(1)-(8), *), * *$ ) und der beiden Formeln:

$\left(\forall v_{0}\right)\left(\forall v_{1}\right)\left(\forall v_{2}\right)\left(\neg p_{1}^{1}\left(v_{1}\right) v\left(p_{4}^{3}\left(v_{0}, v_{1}, v_{2}\right) \longleftrightarrow p_{1}^{1}\left(v_{2}\right)\right)\right)$

$\left(\forall v_{0}\right)\left(\forall v_{1}\right)\left(\forall v_{2}\right)\left(\forall v_{3}\right)\left(\neg p_{2}^{2}\left(v_{3}, v_{1}\right) v\right.$

$\left.\left(p_{4}^{3}\left(v_{0}, v_{1}, v_{2}\right) \longleftrightarrow\left(\exists v_{4}\right)\left(p_{4}^{3}\left(v_{0}, v_{3}, v_{4}\right) \wedge p_{3}^{3}\left(v_{0}, v_{4}, v_{2}\right)\right)\right)\right)$

(3) R entstehe aus $R$, durch Variablenvertauschung und Variablenidentifikation:

$R=\lambda x_{0} \ldots x_{s-1} \cdot R^{\prime}\left(x_{\pi(0)}, \ldots, x_{\pi\left(s^{\prime}-1\right)}\right)$, wobei $\pi$ eine Abbildung von $s^{\prime}$ in $s$ ist. $R^{\prime}$ sei durch $f$ mit $p_{k}^{\prime}$, dargestellt, und $f$ enthalte das zeichen $p_{k}^{s}$ nicht. Dann wird $R$ durch die konjunktion von $f$ mit $\left(\forall v_{0}\right) \ldots\left(\forall v_{s-1}\right)\left(p_{k}^{s}\left(v_{0}, \ldots, v_{s-1}\right) \longleftrightarrow p_{k}^{s^{\prime}}\left(v_{\pi(0)}, \ldots, v_{\pi\left(s^{\prime}-1\right)}\right)\right.$ dargestellt.

(4) Da die Relation $\lambda x \cdot x=0$ und die Nachfolgerrelation darstellbar sind, so auch für jedes $n \in \mathbb{N}$ die Relation $\lambda x \cdot x=n$. Sei diese Relation durch die Formel $f_{1}$ (mit $p_{k}^{1}$ ) dargestellt, und sei $R^{\prime}$ durch $f_{2}$ (mit $p_{k}^{s+1}$ ) dargestellt; weiter sei das Zeichen $p_{k}^{s}$ weder in $f_{1}$ nooh in $f_{2}$ enthalten. Dann wird die Relation $R=\lambda x_{0} \ldots x_{5-1} \cdot R \cdot\left(x_{0}, \ldots, x_{s-1}, n\right)$ durch die Konjunktion von $f_{1}, f_{2}$ und 
$\left(\forall v_{0}\right) \ldots\left(\forall v_{s-1}\right)\left(p_{k}^{s}\left(v_{0}, \ldots, v_{s-1}\right) \longleftrightarrow\left(\exists v_{s}\right)\left(p_{k}^{s+1}\left(v_{0}, \ldots, v_{s}\right) \wedge p_{k}^{1},\left(v_{s}\right)\right)\right)$

dargestelit, sofern nur Modelle der Mächtigkeit grösser als $n$ betrachtet werden. Die endlich vielen anderen fälle lassen sich aber gesondert behandeln.

(5) Ist $R$ eine Boolesche Verknüpfung darstellbarer Formeln, so ist sie klarerweise auch darstellbar.

(6) $R$ ' sei durch $f\left(\right.$ mit $p_{k}^{s}$ ) dargestellt, und $f$ enthalte das Zeichen $p_{k}^{s}$ nicht. Die Relation $R_{1}=\lambda x_{0} \ldots x_{s-1} \cdot\left(\exists x_{s}<x_{s-1}\right) R^{\prime}\left(x_{0}, \ldots, x_{s-2}, x_{s}\right)$ wird durch die Konjunktion von $f$ und $\left(\forall v_{0}\right) \ldots\left(\forall v_{s-1}\right)\left(p_{k}^{s}\left(v_{0}, \ldots, v_{s-1}\right) \longleftrightarrow\left(\exists v_{s}\right)\left(p_{0}^{2}\left(v_{s}, v_{s-1}\right) \wedge\right.\right.$ $\left.\left.P_{k}^{s},\left(v_{0}, \ldots, v_{s}-2, v_{5}\right)\right)\right\}$ dargestellt, und die Relation $R_{2}=\lambda x_{0} \ldots x_{s-1} \cdot\left(\forall x_{s}<x_{s-1}\right) R^{\prime}\left(x_{0}, \ldots, x_{s-2}, x_{s}\right)$ analog durch die Kanjunktion von $f$ und $\left(\forall v_{0}\right) \ldots\left(\forall v_{s-1}\right)\left(p_{k}^{s}\left(v_{0}, \ldots, v_{s-1}\right) \longleftrightarrow\left(\forall v_{s}\right)\left(\neg p_{0}^{2}\left(v_{s}, v_{s-1}\right) v\right.\right.$ $\left.\left.p_{k}^{s},\left(v_{0}, \ldots, v_{s-2}, v_{s}\right)\right)\right)$.

Korollar 2.1 Jede konstruktiv arithmetische Menge von positiven Zahlen ist ein Spektrum.

Beweis Die Menge $M^{\prime}=\{n \mid n+1 \in M\}$ ist genau dann kanstruktiv arithmetisch, wenn M konstruktiv arithmetisch ist. $M$ ' sei durch $f$ mit $p_{k}^{1}$ dargestellt. Das Spektrum von $\left(f \wedge\left(\exists v_{0}\right)\left(p_{k}^{1}\left(v_{0}\right) \wedge\left(\forall v_{1}\right) \neg p_{0}^{2}\left(v_{0}, v_{1}\right)\right)\right)$ ist dann $M$.

Korollar 2.2 $\{f \mid$ Spektrum $(f) \neq \emptyset\}$ hat den maximalen Unentscheidbarkeitsgrad unter den rekursiv aufzählbaren Mengen.

Beweis Nach (Smulyyan 1961, Theorem 8, Seite 89) gibt es eine konstruktiv arithmetische Relation M derart, dass es für jede rekursiv aufzählbare Menge $E$ ein $i \in \mathbb{N}$ gibt mit $E=E_{i}$, wobei

weiter gilt

$$
x \in E_{i} \Longleftrightarrow(\exists y) M(i, x, y) \text {; }
$$

$f^{\prime}$ stelle M mit $p_{k}^{3}$ dar, und $f_{i}^{\prime}$ stelle $\lambda x \cdot x=i$ mit $p_{k}^{1}$, dar. Sei $f_{i}$ die Forme 1

$$
\begin{gathered}
\left(\exists v_{0}\right)\left(\exists v_{1}\right)\left(\exists v_{2}\right)\left(\left(\left(f_{i}^{\prime} \wedge p_{k}^{1},\left(v_{0}\right)\right) \wedge\left(f^{\prime} \wedge p_{k}^{3}\left(v_{0}, v_{1}, v_{2}\right)\right)\right) \wedge\right. \\
\left.\left(\left(\forall v_{3}\right) \neg p_{0}^{2}\left(v_{2}, v_{3}\right) \wedge p_{1}^{1}\left(v_{1}\right)\right)\right) .
\end{gathered}
$$


Die Menge $\left\{i \mid 0 \in E_{i}\right\}$, welche maximalen Unentscheidbarkeitsgrad unter den rekursiv aufzählbaren Mengen hat, ist dann auf Grund der Zuordnung $i \longmapsto f_{i} 1-1$ reduzibel auf $\{f \mid$ Spektrum(f) $\neq \downarrow\}$. Da die Relation $\lambda \times f \cdot x \in$ Spektrum(f) rekursiv ist, so ist die erwähnte Menge rekursiv aufzählbar, und damit ist alles bewiesen.

Korollar 2.3 $\{f \mid$ Spektrum( $f$ ) unendlich $\}$ hat den maximalen Unentscheidbarkeitsgrad unter den $\Pi \Sigma$-Mengen.

Beweis Analog; $p_{1}^{1}\left(v_{1}\right)$ durch $\left(\forall v_{3}\right)\left(\neg p_{0}^{2}\left(v_{3}, v_{2}\right) v \neg p_{k}^{3}\left(v_{0}, v_{1}, v_{3}\right)\right)$ ersetzen.

Korollar 2.4 Für jede rekursive Funktion gibt es ein Spektrum, dessen Aufzählungsfunktion stärker als diese Funktion wächst.

Beweis Nach (Smullyan 1961, Theorem 9, Seite 91) gibt es kanstruktiv arithmetische Relationen $T$ und $U$ derart, dass es für jede rekursive Funktion $f$ ein $i \in \mathbb{N}$ gibt mit $z=f(x) \Longleftrightarrow U(\mu y T(i, x, y), z)$; weiter gelten $U(y, z) \Longrightarrow y>z$ und $T(i, x, y) \Longrightarrow y>i \wedge y>x$. Der Rest verläuft analog.

\section{Spektren und Turingmaschinen}

Durch die dyadische Kodierung - welche der Zahl 0 das leere Wort $\varnothing$ und der Zahl $n=\sum_{j=0}^{m} n_{j} 2^{j}\left(n_{j} \in\{1,2\}\right)$ das Wort $\bar{n}=-b_{n_{0}} \ldots b_{n_{m}}$ zuardnet, und somit eine bijektive Abbildung von $\mathbb{N}$ auf $\left\{b_{1}, b_{2}\right\}$ ist - lässt sich jede Klassifizierung der Teilmengen von $\left\{b_{1}, b_{2}\right\}^{*}$ auf die Teilmengen von $\mathbb{N}$ kanonisch erweitern. Insbesondere wird schlechthin gesagt, eine Teilmenge $M$ von $\mathbb{N}$ liege in $P$, falls die Menge $\bar{M}=\{\bar{n} \mid n \in M\}$ in $P$ liegt. Wie in der Einführung angekündigt, wird in diesem Paragraphen bewiesen, dass die Spektren erster Stufe mit denjenigen Mengen M van positiven Zahlen übereinstimmen, welche schliesslich exponentiell berechenbar sind, d.h. für welche es ein $c \in \mathbb{N}$ und eine schliesslich $\lambda \times \cdot 2^{c \times}$-beschränkte nicht -deterministische NTM gibt, welche M akzeptiert. Dieser Satz ist aequivalent mit dem Satz - welcher etwas einfacher zu beweisen ist - dass die Spektren genau diejenigen Mengen $M$ sind, für welche die Menge $M_{1}=\left\{b_{1}^{(n+1)} \mid n+1 \in M\right\}$ in NP liegt, (wobei $b_{1}^{(n+1)}=\frac{b_{1} \cdots b_{1}}{n+1}$ ).

Zunächst wird der Beweis der trivialeren Richtung skizziert: 
Satz 3 Für jedes Spektrum erster Stufe M liegt die Menge M, in NP.

Beweis M sei das Spektrum der (o.E.d.A.) pränexen Formel $f$, welche $p$ Prädikatszeichen mit Stellenzahl kleiner ader gleich $r$ enthält. Die Zugehörigkeit von $n+1$ zu M wird am direktesten mit einer NTM $T$ auf $\Sigma_{0}=\left\{b_{0}, b_{1}, b_{2}\right\}$ mit mehreren $r$-dimensionalen Bändern getestet, welche folgendermassen operiert:

Der Input befinde sich auf dem nullten Band. T bewertet zuerst nichtdeterministisch auf den Bändern 1 bis p die p Prädikatszeichen von $f$ in einem $(n+1)-e l e m e n t i g e n$ Bereich. Dies erfordert natürlich nur eine polynomiale Schrittzahl.

$T$ bewertet denn die Formel, indem sie sukzessiv alle Bewertungsmöglichkeiten der Variablen probiert und ihrer Quantifikation entsprechend reagiert; die Bewertung einer Variablen kann in der Form eines Wortes der Länge kleiner als $n+1$ auf einem weiteren Band memoriert werden. Für die Auswertung einer Atomformel werden somit für ein passendes $c_{1}$ höchstens $c_{1} \cdot(n+2)$ Schritte benötigt, und für die Auswertung der Matrix von $f$ also höchstens $c_{2} \cdot(n+2)$ Schritte für ein passendes $c_{2}$. Besteht das Präfix von $f$ aus q Variablen, so sind höchstens $(n+2)^{q}$ Bewertungsversuche nötig. Die Schrittzahl von $T$ ist also polynomial beschränkt. Nach den Reduktionssätzen des ersten Vortrags hängt aber NP nicht vom benützten Maschinentypus ab: Somit ist der Satz bewiesen.

Die im Beweis der anderen Richtung benützte Methode ist derjenigen vom vorigen Paragraphen sehr ähnlich: Die Berechnungen einer NTM werden in einem endlichen - jetzt $(n+1)^{k}$-elementigen - Bereich dargestellt. Der Leser wird wohl auch einen Zusammenhang mit dem Beweis des Satzes von Cook (im zweiten Vortrag) erkennen: Die dort angegebenen aussagenlogischen Formeln sind (im wesentlichen!) die $(n+1)$-Redukte der jetzt angegebenen Formel.

Satz 4 Für jede Menge $M_{1}$ aus NP ist die Menge $M=\left\{n+1 \mid b_{1}^{(n+1)} \in M_{1}\right\}$ ein Spektrum erster Stufe.

Beweis $M_{1}$ liege in NP. Nach Voraussetzung wird $M_{1}$ durch eine schliesslich polynomial-beschränkte NTM T akzeptiert. D.E.d.A. sei $T=\left\langle S_{,} s_{0}, s_{1}, R\right\rangle$ mit $S=\left\{s_{0}, s_{1}, \ldots, s_{q-1}\right\}$ eine NTM auf $\sum_{0}$, welche schliesslich $\lambda x \cdot(x+1)^{r}-(x+1)$-beschränkt ist. Weiter sei $\bar{R}=R U\left\{\left\langle b, s_{i}, b, s_{i}\right\rangle \mid \neg(\exists a)(\exists j)\left(\left\langle b, s_{i}, a, s_{j}\right\rangle \in R\right)\right\}$ und $\bar{T}=\left\langle S, s_{0}, S_{1}, \bar{R}\right\rangle$. 
Der Lesbarkeit halber wurden in den folgenden Formeln die Prädikatszeichen mit $K, A, N, F, L, B_{j}(0 \leq j \leq 2)$ und $S_{j}(0 \leq j<q)$ bezeichnet; $t, u, v$, w bezeichnen $r$-Tupel von Variablen. $\left(f_{1} \wedge f_{2} \wedge \ldots \wedge f_{k}\right)$ ist eine Abkürzung von $\left(\left(\ldots\left(f_{1} \wedge f_{2}\right) \wedge \ldots\right) \wedge f_{k}\right),\left(f_{1} \vee f_{2} \vee \ldots \vee f_{k}\right)$ eine Abkürzung von $\left(\left(\ldots\left(f_{1} \vee f_{2}\right) \vee \ldots\right) \vee f_{k}\right)$ und $\left(f_{1} \wedge \ldots \wedge f_{k} \rightarrow f_{k+1} \vee \ldots \vee f_{k+j}\right)$ eine Abkürzung von $\left(\neg\left(f_{1} \wedge \ldots \wedge f_{k}\right) \vee\left(f_{k+1} \vee \ldots \vee f_{k+j}\right)\right)$; weiter ist $u=v$ eine Abkürzung van $\left(u_{0}=v_{0} \wedge \ldots \wedge u_{\Gamma-1}=v_{r-1}\right)$, wobei $u_{j}$, die $j$-te Komponente des $r-$ Tupels u bezeichnet.

$f$ sei die Konjunktion der folgenden 24 Formeln:

(1) $(\forall u)-K(u, u)$

(2) $\quad(\forall u)(\forall v)(K(u, v) \vee k(v, u) \vee u=v)$

(3) $(\forall u)(\forall v)(\forall w)(K(u, v) \wedge K(v, w) \rightarrow K(u, w))$

(4) ( $\exists u) A(u)$

(5) $(\forall u)(\forall v)(A(u) \rightarrow \neg K(v, u))$

(G) $(\forall u)(\forall v)(N(u, v) \rightarrow K(u, v))$

(7) $(\forall u)(\forall v)(\forall w)(N(u, v) \rightarrow \neg K(u, w) \vee \neg K(w, v))$

(B) $(\forall u)(\exists v)(A(u) \vee N(v, u))$

(9) $\left(\forall v_{0}\right)(\exists u) F\left(v_{0}, u\right)$

(10) $\left(\forall v_{0}\right)\left(\forall v_{1}\right)(\forall u)\left(F\left(v_{0}, u\right) \wedge F\left(v_{1}, u\right) \rightarrow v_{0}=v_{1}\right)$

(11) $\left(\forall v_{0}\right)(\forall u)(\forall v)\left(F\left(v_{0}, u\right) \wedge F\left(v_{0}, v\right) \rightarrow u=v\right)$

(12) $\left(\forall v_{0}\right)\left(\forall v_{1}\right)(\forall u)(\forall v)(\forall w)\left(\exists v_{2}\right)$ $\left(F\left(v_{0}, u\right) \wedge F\left(v_{1}, v\right) \wedge K(u, w) \wedge K(w, v) \rightarrow F\left(v_{2}, w\right)\right)$

(13) $(\forall u) \bigvee_{0 \leq j<q} s_{j}(u)$

(14) $(\forall u) \bigwedge_{0 \leq j<k<q}\left(\neg s_{j}(u) v \neg s_{k}(u)\right)$

(15) $(\forall u)(\exists v) L(u, v)$

(16) $(\forall u)(\forall v)(\forall w)(L(u, v) \wedge L(u, w) \rightarrow v=w)$

(17) $(\forall u)(\forall v) \bigvee_{0 \leq j \leq 2} B_{j}(u, v)$

(18) $(\forall u)(\forall v) \bigwedge_{0 \leq j<k \leq 2}\left(\neg \theta_{j}(u, v) v \neg B_{k}(u, v)\right)$

(19) $(\forall u)(\exists v)(\forall w)\left(A(u) \rightarrow\left(s_{0}(u) \wedge L(u, v) \wedge\left(\exists v_{0}\right) F\left(v_{0}, v\right)\right.\right.$

$$
\left.\wedge\left(K(w, v) \rightarrow \neg\left(\exists v_{0}\right) F\left(v_{0}, w\right)\right)\right)
$$

(20) $(\forall u)(\forall v)\left(A(u) \wedge\left(\exists v_{0}\right) F\left(v_{0}, v\right) \rightarrow B_{1}(u, v)\right)$

$(21)(\forall u)(\forall v)\left(A(u) \wedge \neg\left(\exists v_{0}\right) F\left(v_{0}, v\right) \rightarrow B_{0}(u, v)\right)$ 


$$
(\forall u)(\forall v)(\forall w)\left(N(u, v) \wedge \neg L(u, w) \rightarrow \bigwedge_{0 \leq j \leq 2}\left(B_{j}(u, w) \rightarrow B_{j}(v, w)\right)\right)
$$

$(\forall t)(\forall u)(\forall v)(\forall w)(L(u, w) \wedge L(v, t) \wedge N(u, v) \rightarrow$

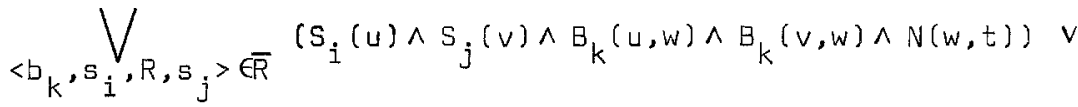

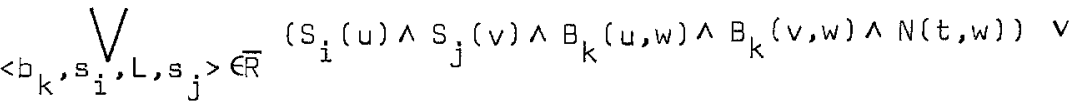

$$
\begin{aligned}
& <b_{k}, s_{i}, b_{\ell}, s_{j}>\bar{R}^{\left.\left(s_{i}(u) \wedge s_{j}(v) \wedge B_{k}(u, w) \wedge t=w \wedge B_{\ell}(v, w)\right)\right)}
\end{aligned}
$$

$(24)$

$$
(\exists u)\left(s_{1}(u) \wedge(\forall v) \neg K(u, v)\right)
$$

(a) Nach Definition gibt es ein $n_{0} \in \mathbb{N}$ derart, dass es eine akzeptierende Berechnung von $T$ der Länge kleiner als $(n+1)^{r}-(n+1)$ mit Input $b_{1}^{(n+1)}$ gibt, falis $n>n$ und $n+1 \in M$. Dies ist aber genau dann der Fall, wenn es eine Berechnung von $\bar{T}$ der Länge $(n+1)^{r}-1$ mit demselben Input gibt, deren letzte Konfiguration den Zustand $s_{1}$ enthält. Sei $\left\langle K_{i}\right\rangle_{i} \in(n+1)^{r}$ mit $k_{i}=\left\langle\left\langle a_{j}^{i}\right\rangle_{j \in \mathbb{Z}}, p^{i}, s^{i}\right\rangle$ eine solche Berechnung.

Ein $(n+1)$-elementiges Modell <M, I> von $f$ kann jetzt folgendermassen definiert werden: $\quad M=n+1=\{0,1, \ldots, n\}$; $I(K)$ sei die lexikographische Ordnung (bez. <) von $M^{r}$ und $I(N)$ die entsprechende Nachfolgerrelation; I(A) sei die charakteristische Relation von $\langle 0, \ldots, 0\rangle$. Dadurch sind die Formeln $(1)-(8)$ erfüllt.

Sei - $\ell$ die Nummer des am weitesten links gelegenen Feldes, welches im Laufe der Berechnung durch den Kopf erreicht wird; da die Länge der entsprechenden Berechnung von T kleiner ist als $(n+1)^{r}-(n+1)$, so ist auch \& kleiner als $(n+1)^{\Gamma}-(n+1)$.

$I(F)$ sei die injektive Abbildung von $M$ nach $M^{r}$, welche dem Element $i$ von $M$ das $(l+i)-t e$ Element von $M^{2}$ bezüglich der lexikographischen Ordnung zuordnet; dies ist auf Grund der obigen Bemerkung möglich. Dadurch sind auch die formeln (9)-(12) erfülit.

$I\left(s_{i}\right)$ sei genau dann vom $j$-ten Element von $M^{\Gamma}$ (bez. der lexikographischen Ordnung) wahr, wenn $s^{j}=s_{i} ; I\left(B_{i}\right) b z w$. $I(L)$ sei genau dann von den $j$-ten und $k$-ten Elementen von $M^{\Gamma}$ (bez. derselben Ordnung), wenn $a_{k+\ell}^{j}=b_{i} b z w \cdot p^{j+l}=k$. Auf Grund der obigen Bemerkung sind dann die Formeln (13)-(18) automatisch erfüllt. (19)-(21) sind erfüllt, weil die Berechnung $\left\langle K_{i}\right\rangle_{i} \in(n+1) r$ den Input $b_{1}^{(n+1)}$ hat, und (22)-(23), weil $K_{i+1}$ Folgekonfiguration von $K_{i}$ ist. Endlich ist (24) erfültt, weil $\bar{T}$ zum Zustand $s_{1}$ gelangt. 
Somit liegt $n+1$ im Spektrum von $f$, falls $n>n_{0}$ und $n+1 \in M$.

(b) Umgekehrt gehöre $n+1$ zum Spektrum von $f$. Dann gibt es ein Modell $\langle M$, I> von f mit $(n+1)$-elementigem $M$. Wegen $(1)-(3)$ ist $I(K)$ eine totale ordnung von $M^{2}$; wegen (4)-(5) gilt $I(A)$ nur für deren erstes Element; wegen (6) $-(8)$ ist $I(N)$ die Nachfolgerelation bezüglich $I(K)$; schliesslich ist wegen (9)-(12) I(F) eine bijektive Abbildung von $M$ auf ein Segment von $M^{r}$ bezüglich $I(K)$.

Sei $m_{0}, m_{1}, \ldots, m_{(n+1)^{r}-1}$ die durch $I(K)$ geordnete Abzählung von $M^{r}$. Nach (13)-(14) gibt es zu jedem $j \in(n+1)^{r}$ genau ein $i(0 \leq i<q)$ mit $I\left(s_{i}\right)\left(m_{j}\right)$ und nach (15)-(16) genau ein $k \in(n+1)^{\Gamma}$ mit $I(L)\left(m_{j}, m_{k}\right)$, sowie zu jedem $j, k \in(n+1)^{r}$ genau sin $i(0 \leq i<2)$ mit $I\left(B_{i}\right)\left(m_{j}, m_{k}\right)$. Sei weiter $\ell$ die kleinste Zahl mit $m_{l} \in I(F)(M)$.

Nun sei für $i \in(n+1)^{r} K_{i}=\left\langle\left\langle a_{j}^{i}\right\rangle_{j \in Z^{\prime}} p^{i}, s^{i}\right\rangle$ durch folgende Gleichungen definiert:

$$
\begin{aligned}
& a_{j}^{i}=\left\{\begin{aligned}
b_{k} & \text { falls } I\left(\theta_{k}\right)\left(m_{j}, m_{j+l}\right) \\
& \text { und }-\ell \leq j<(n+1) r_{-l}, \\
b_{0} & \text { sonst; }
\end{aligned}\right. \\
& P^{i}=j \quad \text { falls } I(L)\left(m_{i}, m_{j+\ell}\right) \text {; } \\
& s^{i}=s_{j} \quad \text { falls } I\left(s_{j}\right)\left(m_{i}\right) \text {; }
\end{aligned}
$$

dies ist auf Grund der vorigen Bemerkung eine vernünftige Definition. Nach (19)-(21) gilt insbesondere:

$$
\begin{aligned}
& a_{j}^{0}=\left\{\begin{array}{l}
b_{1} \text { falls } 0 \leq j \leq n, \\
b_{0} \text { sonst; }
\end{array}\right. \\
& p^{0}=0 ; \\
& s^{0}=\quad s_{0} ;
\end{aligned}
$$

also ist $K_{0}$ die Konfiguration zum Input $b_{1}^{(n+1)}$. Nach (22) und (23) ist $\left\langle K_{i}\right\rangle_{i \in(n+1)^{r}}$ eine Berechnung von $\bar{T}$, und nach (24) gilt $5^{(n+1)^{r-1}}=s_{1}$. Dann ist aber $\left\langle K_{i}\right\rangle_{i} \epsilon_{h+1}$ eine akzeptierende Berechnung von $T$ mit dem Input $b_{1}^{(n+1)}$, d.h. $b_{1}^{(n+1)}$ liegt in $M_{1}$; folglich liegt $n+1$ in $M .\left(h<(n+1)^{r}\right)$

(c) Somit ist gezeigt, dass oberhalb $n_{0}$ die Menge M mit dem Spektrum von $f$ zusammenfällt. Daraus folgt leicht, dass auch $M$ ein spektrum ist: $M$ ist nämlich das Spektrum der Formel $\left(f f_{1}\right) \vee f_{2}$, wobei $f_{1}$ die Gleichheitsformel ist, deren koendliches spektrum aus den Zahlen grösser als $\mathrm{n}_{0}$ besteht und $f_{2}$ die Gleichheitsformel ist, deren endiches spektrum aus 
den Zahlen kleiner oder gleich $n_{0}$ besteht, welche zu M gehören.

Korollar 4.1 Eine Menge M von positiven Zahlen ist genau dann ein Spektrum erster Stufe, wenn $M_{1}=\left\{b_{1}^{(n+1)} \mid n+1 \in M\right\}$ in NP liegt.

Beweis Dies ist eine direkte Folgerung aus den Sätzen 3 und 4.

Korallar 4.2 Eine Menge M von positiven Zahlen ist genau dann ein Spektrum erster Stufe, wenn es eine schliesslich exponentiell-beschränkte ( $d . h$. durch $\lambda x \cdot 2^{\infty x}$ für ein c ) nicht-deterministische NTM gibt, welche $\bar{M}=\{\overline{n+1} \mid n+1 \in M\}$ akzeptiert.

Beweis Da $\operatorname{lh}(\overline{n+1})=\left\lfloor\log _{2}(n+2)\right\rfloor$ gilt

$$
2^{c l h(\overline{n+1})} \leq(n+2)^{c}<2^{c(1+1 h(\overline{n+1}))} \leq 2^{2 \operatorname{clh}(\overline{n+1})} ;
$$

eine Funktion ist also genau dann polynomial in $n+1=\ln \left(b_{1}^{(n+1)}\right)$, wenn sie exponentiell in 1 h $(\overline{n+1})$ ist.

Weiter ist die Zuordnung $\overline{n+1} \mapsto b_{1}^{(n+1)}$ (durch wiederholtes Verdoppeln) in exponentieller Zeit berechenbar auf einer mehrköpfigen TM; ebenfalls ist die Zuordnung $b_{1}^{(n+1)} \rightarrow(\overline{n+1})$ (durch wiederholtes Halbieren) in der Zeit $c(n+1)$ für ein passendes c auf einer-mehrköpfigen TM berechenbar, also in $\Pi$.

Folglich gibt es genau dann eine schliesslich exponentiell-beschränkte NTM, welche $\bar{M}$ akzeptiert, wenn es eine schliesslich polynomial-beschränkte NTM gibt, welche $M_{1}$ akzeptiert.

Analog lassen sich die beiden nächsten Korollare beweisen: Korollar 4.3 Ist NP = P, so sind die Spektren erster Stufe genau die Mengen von positiven Zahlen, welche durch schliesslich exponentiellbeschränkte deterministische TM akzeptiert werden.

Korollar 4.4 Ist NP gegenüber Komplementbildung abgeschlossen, so ist auch die Klasse der Spektren erster Stufe gegenüber Komplementbildung abgeschlossen.

Korollar 4.5 Ist $\bar{M}$ kontext-sensitiv, so sind beide Mengen $\{n+1 \mid \overline{n+1} \in \bar{M}\}$ und $\{n+1 \mid \overline{n+1} \notin \bar{M}\}$ Spektren erster stufe. 
Beweis Bekanntlich sind die kontext-sensitiven Mengen genau die Mengen, welche durch NTM akzeptiert werden, die mit linearem Raum operieren (Kuroda 1964 und Landweber 1963); diese Mengen sowie ihre Komplemente werden durch schliesslich exponentiell-beschränkte deterministische TM akzeptiert.

Korollar 4.6 Jede Menge, deren charakteristische Funktion in der Klasse $\varepsilon^{2}$ von Grzegorczyk liegt, ist ein Spektrum erster Stufe.

Beweis Diese Mengen sind nämlich genaw diejenigen, welche durch TM akzeptiert werden, die mit linearem Raum operieren (Ritchie 1963).

\section{Projektive Klassen und NP}

Gemäss Korollar 4.1 besteht eine direkte Verbindung zwischen den Spektren erster Stufe und den Mengen aus $\left\{b_{1}\right\}^{*}$, welche in NP liegen. Da man aber auf $\left\{b_{1}, b_{2}\right\}^{*}$ auch Teilmengen einer n-elementigen Menge mit Wörtern der Länge $n$ darstellen kann, entsteht auf natürliche Weise die Frage, ob die Mengen aus NP nicht noch enger mit Modellklassen als mit Spektren im Zusammenhang stehen.

Der nicht-deterministische Charakter einer Berechnung entsprach im vorigen Paragraphen der Bewertung eines Prëdikatszeichens: Also müssen wohl projektive Klassen (manchmal pseudo-elementare genannt) betrachtet werden. Wäre jede solche Klasse zugelassen, so wäre (wie bei den Spektren) jede Teilmenge der natürlichen Zahlen darstellbar. Deshalb werden nur endlich axiomatisierbare Klassen betrachtet; natürlich kommen für die Charakterisierung nur endliche Strukturen von endlichem Typus in Frage, d.h. Strukturen mit endich vielen endlichstelligen Relationen (oder Funktionen) auf einem endichen Träger.

Weil die Wörter aus $\left\{b_{1}, b_{2}\right\}$ * Folgen sind, müssen bei der Darstellung die Permutationen des Trägers ausgeschaltet werden: Deshalb werden ausschliesslich geordnete Modelle betrachtet. Ist $R$ eine s-stellige Relation einer n-elementigen geordneten Struktur, so ist ihre kodierung das Wort $w$ der Länge $n^{s}$ mit der Eigenschaft:

$$
R\left(i_{1} \ldots i_{s}\right) \longleftrightarrow(w) \sum_{k=1}^{s} i_{k} n^{k-1}=b_{2} ;
$$

Funktionen können durch ihren Graphen dargestellt werden. Die Kodierung der Relationen $R_{1} \ldots . R_{k}$ ist die Concatenation $w_{1} \ldots w_{k}$ der Kodierungen 
$w_{1}, \ldots, w_{k}$ van $R_{1}, \ldots, R_{k}$.

Der folgende Satz entspricht dem Satz 3 , und wird analog bewiesen:

Satz 5 Zu jeder Formel erster Stufe $f$ mit den (unter anderen) Prädikatszeichen $P_{i} s_{1}, \ldots, P_{i}^{s_{k}}$ gibt es eine Menge $L$ aus NP derert, dass die Kodierung von $I\left(p_{i}^{s_{1}}\right), \ldots I\left(p_{i_{k}}^{s_{k}}\right)$ genau dann in L liegt, wenn es ein Modell<M, I'> von f gibt, mit $I^{\prime}\left(p_{i_{1}}^{s_{1}}\right)=I\left(p_{i_{1}}^{s_{1}}\right), \ldots, I^{\prime}\left(p_{i_{k}}^{s_{k}}\right)=I\left(p_{i_{k}}^{s_{k}}\right)$.

Beweisskizze Zu f gibt es eine NTM $T$, welche folgendermassen operiert: (1) Aus dem Input w der Länge $\mathrm{lh}(\mathrm{w})$ - welcher eventuell eine Kodierung van Relationen ist - wird zunächst $n$ mit $\sum_{j=1}^{k} n^{s}{ }^{s}=1 h(w)$ berechnet. Dafür wird n nicht-deterministisch gewählt, $\sum_{i=1}^{k} n^{5} j$ berechnet, und mit $1 h(w)$ verglichen. Sind beide Zahlen versichieden, so stellt $T$ ab, sonst geht sie zu (2) über.

(2) Die Kodierungen der einzelnen Prädikate werden auf verschiedene Bänder gebracht.

(3) Die weiteren Prädikatszeichen von f werden nicht-deterministisch interpretiert.

(4) Schliesslich wird $f$ auf Erfüllung durch die vorgeschlagene Interpretation getestet:

Für jede der vier Teilberechnungen (1)-(4) wird eine Schrittzahl benötigt, die in $n$ polynomial ist. Da $I h(w) \geq n$ ist also $T$ schliesslich polynomìal-beschränkt.

Die Umkehrung wird mittels folgendem Analogon von Satz 4 erreicht:

Satz 6 Zu jeder Menge $L$ aus NP gibt es eine Formel erster Stufe $f$ mit den - unter anderen - Prädikatszeichen $p_{0}^{2}, p_{1}^{1}, p_{2}^{2}, p_{3}^{1}$ derart, dass das Wort $w \neq \emptyset$ genau dann zu $L$ gehört, wenn es ein geordnetes endliches Modell <M, I> von $f$ gibt, für welches gilt:

(1) $I\left(p_{0}^{2}\right)=(\lambda x y \cdot x<y) / M$

(2) $I\left(p_{1}^{1}\right)=(\lambda x \cdot x=0) / M$

(3) $I\left(p_{2}^{2}\right)=(\lambda x y \cdot y=x+1) \wedge M$

(4) $\overline{\bar{M}}=I h(w)$

(5) Die Kodierung van $I\left(p_{3}^{1}\right)$ ist $w$. 
Beweisskizze $f^{\prime}$ ist die Konjunktion der Formeln (1)-(B) des Lemmas auf Seite 10, der Forme In (1)-(9), (13)-(19) und (22)-(24) aus Satz 4 (Seiten 16-17) und der folgenden Formeln:

$\left(10^{\prime}\right)\left(\forall v_{0}\right)\left(\forall v_{1}\right)(\forall u)(\forall w)\left(F\left(v_{0}, u\right) \wedge F\left(v_{1}, w\right) \rightarrow\left(p_{2}^{2}\left(v_{0}, v_{1}\right) \longleftrightarrow N(u, w)\right)\right)$

$\left(20^{\prime}\right)(\forall u)(\forall v)\left(A(u) \rightarrow\left(B_{0}(u, v) \longleftrightarrow \neg\left(\exists v_{0}\right) F\left(v_{0}, v\right)\right)\right)$

$\left(21^{\prime}\right)(\forall u)\left(\forall v_{0}\right)\left(A(u) \rightarrow\left(p_{3}^{1}\left(v_{0}\right) \longleftrightarrow(\exists v)\left(F\left(v_{0}, v\right) \wedge \theta_{2}(u, v)\right)\right)\right)$

Dadurch wird erzwungen, dass der Input der Berechnung die Kodierung von $I\left(p_{3}^{1}\right)$ ist. Der Rest des Beweises verläuft ganz analog.

Korollar 6.1 Die Mengen von nicht-leeren wörtern aus $\left\{b_{1}, b_{2}\right\}^{*}$, welche in NP liegen, sind genau die Kodierungsmengen der endlichen Strukturen der endlich axiomatisierbaren projektiven Klassen von endlichem Typus.

Beweis Unmittelbar aus Satz 5 und Satz 6.

Korollar 6.2 NP ist genau dann gegenüber Komplementbildung abgeschlossen, wenn es zu jeder endlich axiomatisierbaren projektiven Klasse K von endlichem Typus eine endlich axiomatisierbare projektive klasse vom selben Typus gibt, deren endliche Strukturen genau diejenigen sind, die nicht in K liegen.

\section{Ausblicke auf Spektren höherer Stufe}

Das Spektrumproblem lässt sich für die Formeln höherer Stufe der Typentheorie ohne weiteres veraligemeinern - zumindest für natürliche Zahlen. Die Einschränkung auf $\mathbb{N}$ ist hier nicht in der gleichen Weise wie für die erste stufe berechtigt, und vermeidet eher mengentheoretische Schwierigkeiten: Sind allgemeine Modelle zugelassen (d.h. Modelle, wo die Interpretation der Potenzmenge nicht die volle Potenzmenge zu sein braucht), so gelten zwar immer noch der Kompaktheitssatz und der Satz von Löwenheim; das ist nicht mehr der Fall, wenn nur Standardmodelle zugelassen sind. Leider ist aber dann die klasse der gultigen Formeln nicht mehr rekursiv aufzählbar.

Der Darstellungssatz aus dem dritten Paragraphen lässt sich wie folgt verallgemeinern $\left(v g l\right.$. Christen 1974): Sei $f_{0}=\lambda x \cdot x$ und $f_{i+1}=\lambda x \cdot 2^{f_{i}(x)}$. Eine Menge von positiven Zahlen ist genau dann ein 
Spektrum der $(i+1)$-ten Stufe, wenn es eine schliesslich $\lambda x \cdot f_{i+1}(c x)-$ beschränkte nicht-deterministische Turingmaschine gibt, welche sie akzeptiert. Daraus folgt, dass die Klasse $\gamma_{i}{ }^{*}$ von Rödding die Klasse der Relationen ist, die durch deterministische Turingmaschinen im Raum $\lambda x \cdot f_{i}(c x)$ akzeptiert werden, und dass die Mengen von positiven $Z$ ahlen aus $\gamma_{i}$ * Spektren von (i+1)-ter Stufe sind (Rödding und Schwichtenberg 1972). Insbesondere sind die Spektren beliebiger Stufe genau die elementaren $\left(\xi^{3}\right)$ Mengen von positiven Zahlen (Bennett 1962). Weiter folgt auch, dass es zu jedem $i$ ein Spektrum der $(\mathbf{i}+2)$-ten Stufe gibt, das kein Spektrum der ( $i+1)$-ten Ștufe ist (Bennett 1962, in Christen 1974 vereinfacht).

Als weitere Folgerung des Darstellungssatzes lässt sich zeigen, dass es für jedes unendliche Spektrum der Typentheorie mit unendlichem Komplement eine polynomial entscheidbare Menge gibt, welche das Spektrum sowie sein Komplement in je zwei unendliche Mengen teilt (Christen 1974) (Analoges gilt für kontext-sensitive Mengen und Mengen aus NP). Für jedes $i \in \mathbb{N}$ ist also der Verband $\boldsymbol{J}_{i+1} / \mathcal{F}$ dicht, wobei $\boldsymbol{J}_{i+1}$ die klasse der Spektren der $(i+1)$-ten Stufe und $\mathcal{F}$ die Klasse der endlichen Teilmengen von $\mathbb{N}$ ist; insbesandere gibt es weder minimale noch maximale spektren einer gegebenen Stufe - noch minimale oder maximale Mengen aus NP.

Obwahl die Spektren höherer Stufe eine Hierarchie bilden, lassen sie sich alle als Urbilder von Spektren erster Stufe durch ganz einfache Funktionen darstellen: Eine Menge M ist genau dann ein Spektrum (i+1)ter Stufe, falls es ein c und ein Spektrum erster stufe $M_{1}$ derart gibt, dass $M=\left\{n \mid f_{i}\left(n^{C}\right) \in M_{1}\right\}$ (Bennett 1962). Sie sind ebenfalls Urbilder der Mengen aus NP durch die Funktionen $\lambda x \cdot f_{i+1}\left(x^{c}\right)$. Daraus folgt, dass für jedes $i \in \mathbb{N}$ die Klasse der Spektren $(i+2)$-ter Stufe gegenüber Komplementbildung abgeschlossen ist, falls die Klasse der Spektren (i+1)ter Stufe gegenüber Kamplementbildung abgeschlossen ist, und analog, dass die Spektren $(i+2)$-ter Stufe für $c \in \mathbb{N}$ deterministisch $\lambda x \cdot f_{i+2}(c x)-$ berechenbar sind, falls die Spektren ( $i+1)$-ter Stufe deterministisch $\lambda x \cdot f_{i+1}(0 x)$ berechenbar sind. In analoger Weise lassen sich die Spektren der sogenannten "schwachen" ( $i+2)$-ten Stufe (d.h. die Spektren von Formeln, welche eventuell gebundene Prädikatszeichen der (i+1)-ten Stufe, doch keine Prädikatszeichen der ( $i+2)$-ten Stufe enthalten) als Urbilder (durch $\lambda x \cdot f_{i+1}(c x)$ für $c \in \mathbb{N}$ ) der konstruktiv arithmetischen Mengen darstellen (Bennett 1962), von (Rödding und Schwichtenberg 1972) unabhängig wiedergefunden). 
Auf Grund dieser Eigenschaften ist die kombinatorische Komplexität der höheren Klassen in einem gewissen Sinne die gleiche wie diejenige der tieferen Klassen. Vielleicht ist ein Teil der Schwierigkeiten in der Komplexitätstheorie - wie z.B. der manchmal ungeheure Unterschied zwischen den bekannten oberen und unteren Kamplexitätsschranken - auf ein derartiges Phänomen zurückzuführen.

\section{Literatur}

Ackermann, W., Ueber die Hilbertsche Definition der reellen Zahlen, Math. Ann. 99 (1928) 118-133.

Ackermann, W., Solvable Cases of the Decision Problem, North-Holland, Amsterdam 1954.

Asser, G., Das Repräsentantenproblem im Prädikatenkalkül der ersten Stufe mit Identität, $Z$. Math. Logik Grundlagen Math. 1 (1956) 252-263.

Bennett, J., On Spectra, Doctoral Dissertation, Princeton University, 1962 (Microfilm H01: 63-496).

Büchi, J.R., Turing Machines and the Entscheidungsproblem, Math. Ann. 148 (1962) 201-213.

Christen, C.A., Spektren und Klassen elementarer Funktionen, Dissertation 5330, ETH Zürich 1974.

Jones, N.D./Selman, A.L., Turing Machines and the Spectra of Firstorder Formulas, J. Symbolic Logic 39 (1974) 139-150.

Kuroda, S.Y., Classes of languages and linear-bounded automata, Information and Control 7 (1964) 207-223.

Landweber, P., Three theorems on phrase structure grammars of type 1 , Information and Control 6 (1963) 131-137.

Löwenheim, L., Ueber Möglichkeiten im Relativkalkül, Math. Ann. 76 (1915) 137-148.

Pazderski, G., Die Ordnungen, zu denen nur Gruppen mit gegebener Eigenschaft gehören, Arch. Math. 10 (1959) 331-343.

Ritchie, R.W., Classes of predictably computable functions, Trans. AMS 105 (1963) 139-173.

Rödding, D./Schwichtenterg, H., Bemerkungen zum Spektralproblem, Z. Math. Logik Grundlagen Math. 18 (1972) 1-12.

Scholz, H., Ein ungelöstes Problem in der symbolischen Logik, J. Symbolic Logic 17 (1952) 160. 
Smullyan, R., Theory of Formal Systems, Ann. of Math. Studies 47. Princeton 1961.

Trachtentrot, B.A., Névozmojnost algorifma dla problemy razrésimosti na konécyh klassah (Unmöglichkeit eines Algorithmus für das Entscheidungsproblem in endichen Klassen), Doklady Akad. Nauk SSSR 70 (1950) 569-572. 